\title{
TRANSMIT/RECEIVE BEAMFORMER DESIGN AND POWER CONTROL IN MIMO MC-CDMA SYSTEMS
}

\author{
S. C. Chan and S. H. Zhao \\ Department of Electrical and Electronic Engineering \\ The University of Hong Kong, Pokfulam Road, Hong Kong \\ scchan@eee.hku.hk, shzhao@eee.hku.hk
}

\begin{abstract}
In this paper, a joint transmitter and receiver beamformers design algorithm for downlink multiple input multiple output multicarrier code-division multiple access (MIMO MC-CDMA) system is proposed. The algorithm is iterative in nature where the transmitter beamformers and the receiver beamformers are determined alternately. The transmitter beamforming problem with a given receiver beamformer is formulated as a convex programming problem, which can be solved optimally using second order cone programming (SOCP), while the receiver beamforming problem is formulated as a constrained optimization problem with an analytical solution. The convergence of the algorithm is analyzed and the performance of the proposed algorithm is evaluated by computing simulation.
\end{abstract}

\section{INTRODUCTION}

The multicarrier code-division multiple-access (MCCDMA) system is a promising candidate for future wireless communication system. It has received considerable attention recently [1][2][3]. MC-CDMA system combines the advantage of Orthogonal Frequency Division Multiplex (OFDM) modulation and spread spectrum (SS) technique and is able to provide flexible multiple access and frequency diversity in frequency selective fading channels. On the other hand, multiple input and multiple output (MIMO) systems using multiple transmit and multiple receive antennas can achieve very high spectral efficiency in rich multipath environment through spatial diversity [4]. Therefore, MIMO MC-CDMA systems are of great interest in achieving high data rate and multiple access services in wireless channels.

In cellular and personal communication systems, interference among different users is a fundamental limitation to system capacity. The use of multiple transmit and receive antennas with appropriate beamforming offers another freedom to reduce the interferences among users. The design of transmitter and receiver beamformers for different communications systems are the subjects of active research [5]-[7]. In [5], a joint transmit and receive beamforming design for multicarrier MIMO system is developed and a unified framework based on convex programming that can embrace most reasonable criterion was proposed. In [6], the problem of transmitter and receiver design for MC-CDMA systems is considered. Under constraints of given quality of service, analytical transmitter and receiver solutions for the additive white Gaussian noise (AWGN) channels and fading channels are derived to minimize the transmit power. In [7], an MMSE based beamforming algorithm for MC-CDMA system is discussed. The beamvectors are first determined by means of a training sequence and they are updated by a decision-directed technique.

In this paper, a joint transmitter and receiver beamformer design algorithm for downlink MIMO MCCDMA systems is proposed. Under a given quality of service (Qos) in term of the signal to interference plus noise ratio (SINR) of all users, the total transmission power is minimized. The algorithm alternately determines the beamformer weights for the transmitters and receivers, while keeping the values of the other weights fixed. For the transmitter, the design problem is cast as a second order cone programming (SOCP) which can be solved optimally and efficiently using Interior Point (IP) method [8]. Another advantage of the SOCP formulation is that additional linear constraints and convex quadratic constraints can readily be incorporated. For the receiver, the beamformer design problem is formulated as a constrained optimization problem and it yields an analytical solution, which is recognized as the $\mathrm{CMOE}$ receiver.

The paper is organized as follows: the system model and problem formulation are described in section II. Section III is devoted to the beamformers design of the transmitters and receivers. Simulation results are given in Section IV. Finally, conclusions are drawn in Section V.

\section{SYSTEM MODEL AND PROBLEM FORMULATION}

Consider the downlink of a MIMO MC-CDMA system with $N_{t}$ transmit antennas at the base station (BS). Assume that there are $M$ mobile users (MS) and each is communicating with the BS with $N_{r}$ receiver antennas. The channel information is assumed known at both sides. Let $s_{m}[n, k]$ be the message symbol of the $m$-th user at the $n$-th OFDM frame and tone $k, k=1, \ldots K$. For simplicity, we shall assume that the message symbols between different users are uncorrelated and the message symbol $s_{m}[n, k]$ has unit magnitude, i.e. $E\left[s_{m}[n, k] s_{m^{\prime}}[n, k]\right]=\delta\left(m-m^{\prime}\right)$. We will drop the index $n$ in subsequent discussion for simplicity. Let the beamforming weight of $s_{m}[k]$ at the $i$-th transmit antenna be $t_{m, i}[k]$. The $k$-th tone signal transmitted at the $i$-th transmit antenna is then given by 


$$
x_{i}[k]=\sum_{m=1}^{M} t_{m, i}[k] s_{m}[k],
$$

and

$$
\boldsymbol{x}[k]=\left[x_{1}[k], \ldots, x_{N_{t}}[k]\right]^{T}=\sum_{m=1}^{M} \boldsymbol{t}_{m}[k] s_{m}[k],
$$

where $\quad \boldsymbol{t}_{m}[k]=\left[t_{m, 1}[k], \ldots, t_{m, N_{t}}[k]\right]^{T}$.

The time-domain signal for the $i$-th antenna is obtained by taking the $K$-point IDFT of the sequence $\left\{x_{i}[k]\right\}$, after inserting the cyclic prefix. Let the $\left(N_{r} \times N_{t}\right)$ channel matrix from the BS to the $m$-th mobile user be $\boldsymbol{h}_{m}[l]$. If the length of the cyclic prefix is larger than the maximum length of all the components in $\boldsymbol{h}_{m}[l]$, then the ISI of each channel can be removed by dropping the cyclic prefix at the receiver side. In the frequency domain, the received signal at the $m$-th mobile is given by:

$$
\boldsymbol{y}_{m}[k]=\boldsymbol{H}_{m}[k] \boldsymbol{x}[k]+\boldsymbol{n}_{m}[k],
$$

where $\boldsymbol{H}_{m}[k]=\sum_{l=0}^{K-1} \boldsymbol{h}_{m}[l] W_{K}^{k k}, W_{K}^{k k}=\exp (-2 \pi l k / K)$, and $\boldsymbol{n}_{m}[k]$ is the additive White Gaussian Noise (AWGN) vector with zero mean and covariance $\sigma_{m}^{2}$. Let $\boldsymbol{r}_{m}^{+}[k]$ be the beamforming weight of the $m$-th mobile for the $m$-th user at the $k$-th carrier, the received signal at the $m$-th mobile after beamforming at the receiver should be:

$$
\hat{s}_{m}[k]=\boldsymbol{r}_{m}^{+}[k] \boldsymbol{y}_{m}[k] .
$$

Here, "+" denotes the conjugate transpose operation. Substituting (2) into (3), we get

$$
\begin{aligned}
\hat{s}_{m}[k]= & \boldsymbol{r}_{m}^{+}[k] \boldsymbol{H}_{m}[k] \boldsymbol{t}_{m}[k] s_{m}[k] \\
& +\left(\boldsymbol{r}_{m}^{+}[k] \boldsymbol{H}_{m}[k] \sum_{n \neq m} \boldsymbol{t}_{n}[k] s_{n}[k]+\boldsymbol{r}_{m}^{+} \boldsymbol{n}_{m}[k]\right) .
\end{aligned}
$$

It can be seen that the first term $\boldsymbol{r}_{m}^{+}[k] \boldsymbol{H}_{m}[k] \boldsymbol{t}_{m}[k] s_{m}[k]$ is the desired transmitted signal, and the second term $\eta_{m}(k)=\boldsymbol{r}_{m}^{+}[k] \boldsymbol{H}_{m}[k] \sum_{n=m} \boldsymbol{t}_{n}[k] s_{n}[k]+\boldsymbol{r}_{m}^{+} \boldsymbol{n}_{m}[k]$ is the interferences from other users plus channel noises. Since $s_{m}[k], s_{n}[k]$, and $n_{m}[k]$ are uncorrelated, the received signal power is

$$
\begin{aligned}
& E\left[s_{m}^{+}[k] s_{m}[k]\right]=E\left[\boldsymbol{r}_{m}^{+}[k] \boldsymbol{H}_{m}[k] \boldsymbol{t}_{m}[k] s_{m}[k] \cdot\left(\boldsymbol{r}_{m}^{+}[k] \boldsymbol{H}_{m}[k] \boldsymbol{t}_{m}[k] s_{m}[k]\right)^{+}\right] \\
& =\boldsymbol{r}_{m}^{+}[k] \boldsymbol{H}_{m}[k] \boldsymbol{t}_{m}[k] \cdot E\left[s_{m}[k] \cdot s_{m}[k]\right] \cdot\left(\boldsymbol{r}_{m}^{+}[k] \boldsymbol{H}_{m}[k] \boldsymbol{t}_{m}[k]\right)^{+} \\
& =\left\|\boldsymbol{r}_{m}^{+}[k] \boldsymbol{H}_{m}[k] \boldsymbol{t}_{m}[k]\right\|_{2}^{2} .
\end{aligned}
$$

Similarly, the interference plus noise power is

$$
E\left[\left\|\eta_{m}(k)\right\|_{2}^{2}\right]=\sum_{n \neq m}\left\|\boldsymbol{r}_{m}^{+}[k] \boldsymbol{H}_{m}[k] \boldsymbol{t}_{n}[k]\right\|_{2}^{2}+\boldsymbol{r}_{m}^{+}[k] \boldsymbol{R}_{\eta_{m}} \boldsymbol{r}_{m}[k] .
$$

For white Gaussian noise, $\boldsymbol{R}_{n_{m}}=\sigma_{m}^{2} \boldsymbol{I}$. The SINR for the $k$-th tone is then given by:

$$
\operatorname{SINR}_{m}[k]=\frac{\left\|\boldsymbol{r}_{m}^{+}[k] \boldsymbol{H}_{m}[k] \boldsymbol{t}_{m}[k]\right\|_{2}^{2}}{\sum_{n \neq m}\left\|\boldsymbol{r}_{m}^{+}[k] \boldsymbol{H}_{m}[k] \boldsymbol{t}_{n}[k]\right\|_{2}^{2}+\boldsymbol{r}_{m}^{+}[k] \boldsymbol{R}_{n_{m}} \boldsymbol{r}_{m}[k]} .
$$

For MIMO MC-CDMA, a signal message is sent through several sub-carriers or tones using a spreading code. That is, $s_{m}[k]=c_{m}[k] s_{m}$, where $c_{m}[k], k=0, \ldots, K-1$ is the spreading code of the $m$-th user. The output of the $m$-th receiver is

$$
\hat{s}_{m}=\sum_{k=0}^{K-1} y_{m}[k],
$$

where $\quad \boldsymbol{y}_{m}[k]=\boldsymbol{r}_{m}^{+}[k]\left\{\boldsymbol{H}_{m}[k]\left(\boldsymbol{t}_{m}[k] c_{m}[k]\right) s_{m}+\boldsymbol{n}_{m}[k]\right\}$.
In matrix form, we have

$$
\hat{s}_{m}=\boldsymbol{r}_{m}^{+} \boldsymbol{H}_{m} \boldsymbol{t}_{m} s_{m}+\boldsymbol{r}_{\boldsymbol{m}}^{+} \boldsymbol{H}_{m}\left(\sum_{n \neq m} \boldsymbol{t}_{n} s_{n}\right)+\boldsymbol{r}_{m}^{+} \boldsymbol{n}_{m},
$$

where $\boldsymbol{r}_{m}^{+}=\left[\boldsymbol{r}_{m}^{+}[0], \ldots, \boldsymbol{r}_{m}^{+}[K-1]\right]$,

$$
\begin{aligned}
& \boldsymbol{H}_{m}=\operatorname{diag}\left\{\boldsymbol{H}_{m}[0], \ldots, \boldsymbol{H}_{m}[K-1]\right\}, \\
& \left.\boldsymbol{t}_{m}=\left[\left(\boldsymbol{t}_{m}[0] c_{m}[0]\right)^{T}, \ldots, \boldsymbol{t}_{m}[K-1] c_{m}[K-1]\right)^{T}\right]^{T}, \\
& \text { and } \boldsymbol{n}_{m}=\left[\boldsymbol{n}_{m}^{T}[0], \ldots, \boldsymbol{n}_{m}^{T}[K-1]\right]^{T} .
\end{aligned}
$$

From (9) we can see that the estimation error $e_{m}$ is

$\hat{s}_{m}-s_{m}=\left(\boldsymbol{r}_{m}^{+} \boldsymbol{H}_{m} \boldsymbol{t}_{m}-1\right) s_{m}+\boldsymbol{r}_{m}^{+}\left[\boldsymbol{H}_{m}\left(\sum_{n \neq m} \boldsymbol{t}_{n} s_{n}\right)+\boldsymbol{n}_{m}\right]$.

If the additive noise and interference $n i_{m}=\boldsymbol{r}_{m}^{+}\left[\boldsymbol{H}_{m}\left(\sum_{n \neq m} \boldsymbol{t}_{n} s_{n}\right)+\boldsymbol{n}_{m}\right] \quad$ is Gaussian, $\quad\left(\boldsymbol{r}_{m}, \boldsymbol{t}_{m}\right)$, $m=1, \ldots, M$, should be chosen such that

i) $\boldsymbol{r}_{m}^{+} \boldsymbol{H}_{m} \boldsymbol{t}_{m}=1, m=1, \ldots, M$, and

ii) minimize $\sigma_{n_{m}}^{2}=E\left[\left\|\boldsymbol{r}_{m}^{+}\left[\boldsymbol{H}_{m}\left(\sum_{m=m} \boldsymbol{t}_{n} s_{n}\right)+\boldsymbol{n}_{m}\right]\right\|_{2}^{2}\right]$ by some criterion.

Since the components of $s_{m}$ in $\hat{s}_{m}$ should be equal to one by the constraints in i), we can choose to minimize $\sigma_{n i_{m}}^{2}$ subject to the constraints $\boldsymbol{r}_{m}^{+} \boldsymbol{H}_{m} \boldsymbol{t}_{m}=1$. This yields the CMOE receiver. The solution will be given late in section III.B.

Due to near-far problem, we would like to control the radiated power from the $\mathrm{BS}$ such that the SINR is maintained above a certain value for all users. Our design objective is to jointly design the transmitter and receiver beamforming weights so that the total transmit power at the base station is minimized under the constraint of satisfying all users' QoS, e.g SINR. The design objective can be cast into the following optimization problem:

$$
\begin{aligned}
& \operatorname{minimize} \sum_{m=1}^{M}\left\|\boldsymbol{t}_{m}\right\|_{2}^{2} \\
& \text { subject to } \operatorname{SINR} R_{m}=\frac{\left\|\boldsymbol{r}_{m}^{+} \boldsymbol{H}_{m} \boldsymbol{t}_{m}\right\|_{2}^{2}}{\sum_{n \neq m}\left\|\boldsymbol{r}_{m}^{+} \boldsymbol{H}_{m} \boldsymbol{t}_{n}\right\|_{2}^{2}+\boldsymbol{r}_{m}^{+} \boldsymbol{R}_{n_{m}} \boldsymbol{r}_{m}} \geq \gamma_{m}, \\
& m=1, \ldots, M, \text { where } \gamma_{m} \text { is a given constant. }
\end{aligned}
$$

\section{Transmitter And Receiver BeAmformer Design}

\section{A. Transmitter Beamformer Optimization}

To design the transmit beamformer, we assume that the receiver beamformer is fixed. The objective we try to minimize is the transmit power of the BS, which is the sum of the Euclidean norm of the transmitter beamvectors $\boldsymbol{t}_{n}$. First of all, (11) can be reformulated as

$$
\min _{t_{1}, \ldots, t_{M}} \delta \quad \text { subject to } \delta \geq \sum_{m=1}^{M}\left\|\boldsymbol{t}_{m}\right\|_{2}^{2}
$$

and

$$
\left(\gamma_{m}{ }^{-1 / 2} \boldsymbol{r}_{m}^{+} \boldsymbol{H}_{m}\right) \boldsymbol{t}_{m} \geq\left(\sum_{n \neq m}\left\|\boldsymbol{r}_{m}^{+} \boldsymbol{H}_{m} \boldsymbol{t}_{n}\right\|_{2}^{2}+\left\|\hat{\boldsymbol{R}}_{n_{m}} \boldsymbol{r}_{m}\right\|_{2}^{2}\right)^{1 / 2},
$$

where $\quad \hat{R}_{n_{m}}=\sigma_{m} I, m=1, \ldots M$.

An important step is to recognize that (12) can be cast into the following standard SOCP problem: 


$$
\min _{x} c^{T} \boldsymbol{x}
$$

subject to $\boldsymbol{c}^{T} \boldsymbol{x} \geq\|\boldsymbol{A} \boldsymbol{x}\|_{2}$, and $\boldsymbol{b}_{m} \boldsymbol{x} \geq\left\|\boldsymbol{G}_{m}\right\|_{2}, m=1, \ldots M$,

where $\boldsymbol{t}=\left[\boldsymbol{t}_{1}^{T}, \ldots, \boldsymbol{t}_{M}^{T}\right]^{T}, \boldsymbol{x}=\left[\begin{array}{ll}\delta & \boldsymbol{t}^{T}\end{array}\right]^{T}, \boldsymbol{c}=\left[\begin{array}{ll}1 & \boldsymbol{O}_{N_{t} K M}^{T}\end{array}\right]^{T}$, $\boldsymbol{A}=\left[\begin{array}{ll}\boldsymbol{O}_{N_{t} K M} & \boldsymbol{I}_{N_{t} K M}\end{array}\right], \boldsymbol{b}_{m}=\gamma_{m}^{-1 / 2} \boldsymbol{r}_{m}^{+} \boldsymbol{H}_{m} \boldsymbol{E}^{\boldsymbol{m}}, \boldsymbol{G}_{m}=\left[\begin{array}{ll}\boldsymbol{x}^{T} \boldsymbol{F}_{m}^{T} & \boldsymbol{r}_{m}^{T} \hat{\boldsymbol{R}}_{n_{m}}\end{array}\right]^{T}$,

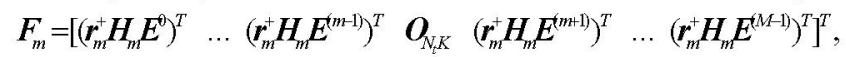
$\boldsymbol{O}_{N}^{T}$ is a $N$ row zero vector, $\boldsymbol{I}_{N}$ is a $N$ by $N$ identity matrix, and $\boldsymbol{E}^{m}$ is a $N_{t} K$ by $N_{t} K M+1$ matrix with the elements: $e_{i, i+m N_{t} K+1}=1$ for $i=1, \ldots N_{t} K$ and zero otherwise. The feasibility of (13) depends on the quality of the channel. If it exits, the optimal solution can be found by the above convex SOCP problem.

\section{B. Receiver Beamformer Optimization}

To reduce the interference, $r_{m}$ should overlap as much as possible with the null space of $\boldsymbol{H}_{m}\left(\sum_{n \neq m} \boldsymbol{t}_{n} s_{n}\right)+\boldsymbol{n}_{m}$. For a given $\boldsymbol{t}_{m}$, the constraint $\boldsymbol{r}_{m}^{+}\left(\boldsymbol{H}_{m} \boldsymbol{t}_{m}\right)=1$ is linear in $\boldsymbol{r}_{m}$ and the objective is to minimize $E\left[\left(e_{m}\right)^{2}\right]$. This gives

$$
\begin{aligned}
& \min _{\boldsymbol{r}_{m}} \boldsymbol{r}_{\boldsymbol{m}}^{+}\left[\boldsymbol{H}_{m}\left(\sum_{n \neq m} \boldsymbol{t}_{n} \boldsymbol{t}_{n}^{+}\right) \boldsymbol{H}_{m}^{+}+\boldsymbol{R}_{m}\right] \boldsymbol{r}_{\boldsymbol{m}} \\
& \text { subject to } \boldsymbol{r}_{\boldsymbol{m}}^{+}\left(\boldsymbol{H}_{m} \boldsymbol{t}_{\boldsymbol{m}}\right)=1 .
\end{aligned}
$$

(14) is a standard problem in constrained optimization, which has the solution:

$$
\boldsymbol{r}_{m}=c_{m}\left[\boldsymbol{H}_{m}\left(\sum_{n \neq m} \boldsymbol{t}_{n} \boldsymbol{t}_{n}^{+}\right) \boldsymbol{H}_{m}^{+}+\boldsymbol{R}_{m}\right]^{-1}\left(\boldsymbol{H}_{m} \boldsymbol{t}_{m}\right),
$$

where $c_{m}=\left[\left(\boldsymbol{H}_{m} \boldsymbol{t}_{m}\right)^{+}\left[\boldsymbol{H}_{m}\left(\sum_{n \neq m} \boldsymbol{t}_{n} \boldsymbol{t}_{n}^{+}\right) \boldsymbol{H}_{m}^{+}+\boldsymbol{R}_{m}\right]^{-1}\left(\boldsymbol{H}_{m} \boldsymbol{t}_{\boldsymbol{m}}\right)\right]^{-1}$. It is clear that the resulting receiver is a CMOE receiver. Note that, the existence of a solution to (15) requires that the matrix $\left[\boldsymbol{H}_{m}\left(\sum_{n \neq m} \boldsymbol{t}_{n} \boldsymbol{t}_{n}^{+}\right) \boldsymbol{H}_{m}^{+}+\boldsymbol{R}_{m}\right]$ be nonsingular. If it is singular, it means that some of the tones or the transmitter beamformers weights are zeros out and not all the information can pass through.

\section{Algorithm Description and Convergence Analysis}

The transmitter and receiver beamformers are determined alternately. At the beginning of the algorithm, the receiver beamvector $\boldsymbol{r}_{m}$ is first initialized to a random value, or other appropriate value if prior information is available. Then, the transmitter beamvector is solved by the SOCP described in section III.A. A feasible transmitter beamvector solution so obtained is denoted by $\boldsymbol{t}_{m}^{(i)}$ where $i$ denotes the $i$-th iteration. Then the receiver beamvector is updated using $t_{m}^{(i)}$ as discussed in sectionIII.B. This process will be repeated until the relative change of total power $\Delta P_{T}$ is small than a certain value.

We now briefly analyze the convergence of the algorithm. Assuming two consecutive feasible solution $\left\{\boldsymbol{t}_{\boldsymbol{m}}^{(i)}, \boldsymbol{r}_{\boldsymbol{m}}^{(i)}\right\}$ and $\left\{\boldsymbol{t}_{\boldsymbol{m}}^{(i+1)}, \boldsymbol{r}_{\boldsymbol{m}}^{(i+1)}\right\}$ for user $m$ is obtained in the $i$-th and $i+1$-th iteration respectively. Note that, $r_{m}^{(i+1)}$ is obtained by (14), then it follows that among all feasible solutions, $\boldsymbol{r}_{m}^{(i+1)}$ maximize $S I N R_{m}$. In other words,

$\frac{\left\|\left(\boldsymbol{r}_{m}^{(i+1)}\right)^{+} \boldsymbol{H}_{m} \boldsymbol{t}_{m}^{(i)}\right\|_{2}^{2}}{\sum_{z \neq m}\left\|\left(\boldsymbol{r}_{m}^{(i+1)}\right)^{+} \boldsymbol{H}_{m} \boldsymbol{t}_{m}^{(i)}\right\|_{2}^{2}+\left(\boldsymbol{r}_{m}^{(i+1)}\right)^{+} \boldsymbol{R}_{h_{m}} \boldsymbol{r}_{m}^{(i+1)}} \geq \frac{\left\|\left(\boldsymbol{r}_{m}^{(i)}\right)^{+} \boldsymbol{H}_{m} \boldsymbol{t}_{m}^{(i)}\right\|_{2}^{2}}{\sum_{n \neq m}\left\|\left(\boldsymbol{r}_{m}^{(i)}\right)^{+} \boldsymbol{H}_{m} \boldsymbol{t}_{m}^{(i)}\right\|_{2}^{2}+\left(\boldsymbol{r}_{m}^{(i)}\right)^{+} \boldsymbol{R}_{h_{m}} \boldsymbol{r}_{m}^{(i)}}$ When $\boldsymbol{r}_{m}^{(i+1)}$ is used to update transmitter beamvector $\boldsymbol{t}_{m}^{(i+1)}$ by (13), it should be able to maintain the given SINR with a lower or equal transmit power than the last iteration. That is:

$$
P_{t}^{(i)}=\sum_{m=1}^{M}\left\|\boldsymbol{t}_{m}^{(i)}\right\|_{2}^{2} \geq \sum_{m=1}^{M}\left\|\boldsymbol{t}_{m}^{(i+1)}\right\|_{2}^{2}=P_{t}^{(i+1)} .
$$

(16) shows that the transmit power in the proposed iterative algorithm is monotonically decreasing. Since the transmit power is lower bounded by zero, the algorithm will converge.

\section{Simulation Results}

In this section, the performance of the proposed algorithm is evaluated using computer simulations. Unless otherwise specified, receive antennas for each user, transmit antennas, and subcarrier number are set to 2, 8, and 16, respectively. For simplicity, all users are assumed has an equal noise power $\sigma^{2}$. As MC-CMDA systems are robust to frequency selective fading, when the number of subcarrier is large enough, each subchannel can be viewed as a flat fading channel. So, all subchannels are modeled as flat fading channels in the simulation. All the design problems were solved by the SeDuMi Matlab Toolbox [12] on a Pentium 4 PC. Design results are plotted in Figures 1 to 4 .

Beamformers design for a 3-user and an 8-user so called "balance system" are first carried out, respectively. In a balance system, all users are assumed to have the same QoS. From equation (7), it can be seen that when the SINR for each user is identical, then due to the interference term, the transmit power should increase linearly with the SINR in order to maintain the given QoS for all users. This is demonstrated in Figure 1. It can also be seen from the result that, when the number of MS increases, interference among users increases accordingly, hence more transmit power is required.

Figure 2 illustrates the design results for a 3-user and an 8-user unbalanced systems, where different users have different QoS. In the design examples, we vary the SINR of one user from $0 \mathrm{~dB}$ to $25 \mathrm{~dB}$, while fixing the SINR of the other users to $0 \mathrm{~dB}$ for the 3 -user system and $10 \mathrm{~dB}$ for the 8 user system, respectively. For the users with fixed SINR, the transmit powers almost remain constant and so does the inference to other users caused by them. Referring to (7), when the user number is large enough, the function approximates a quadric curve, which can be observed in figure 2 for the 8 user sytem.

Figure 3 shows the convergence behavior of the proposed algorithm for a typical channel realization with the receiver beamvectors being randomly initialized. It can be seen from the figure that the design algorithm converges and is not too sensitive to the initial value. 


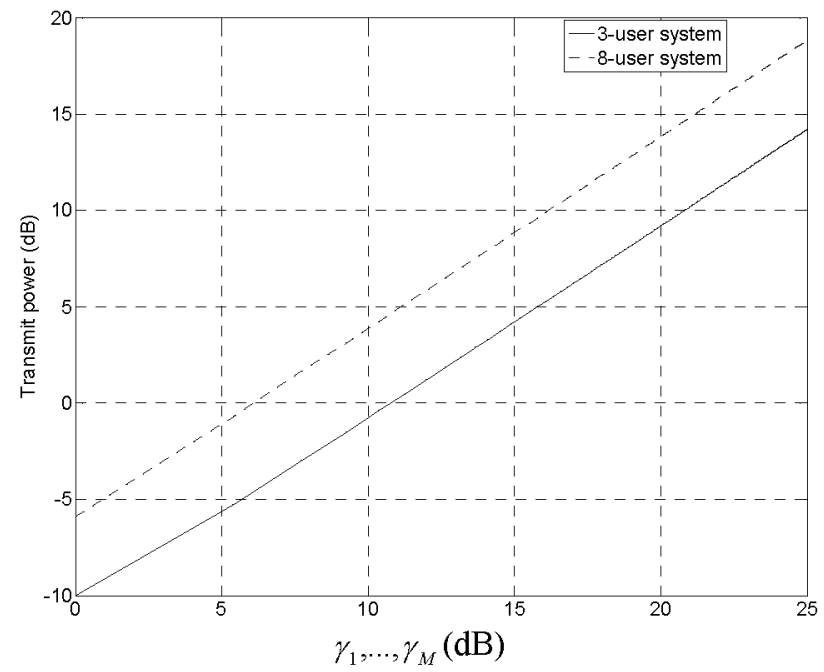

Figure 1. Performance of balance systems with equal SINR, $M=3$ or 8 .

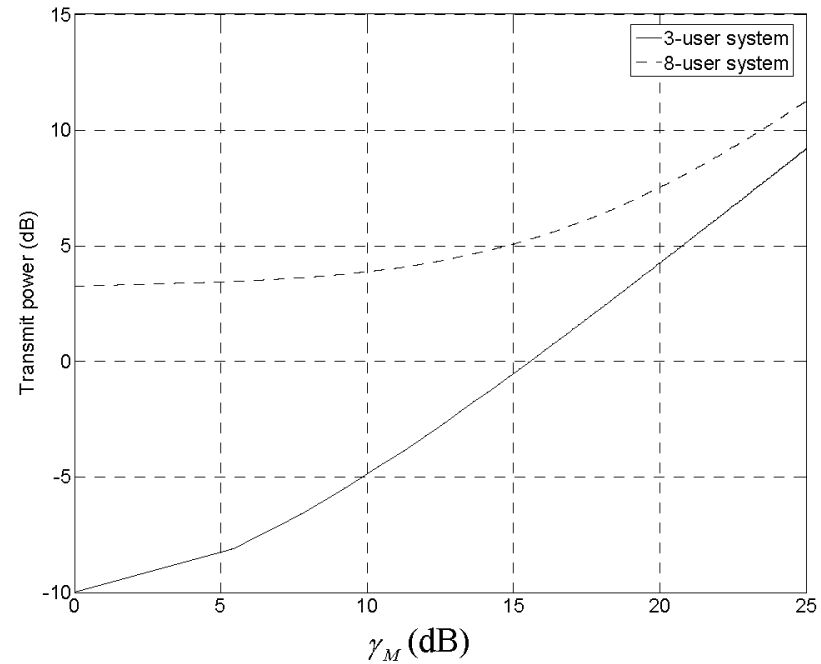

Figure 2. Performance of unbalanced systems: $\gamma_{1}=\gamma_{2}=\gamma_{3}=0 \mathrm{~dB}$ for a 3-user system, and $\gamma_{1}=\ldots=\gamma_{7}=10 \mathrm{~dB}$ for a 8-user system.

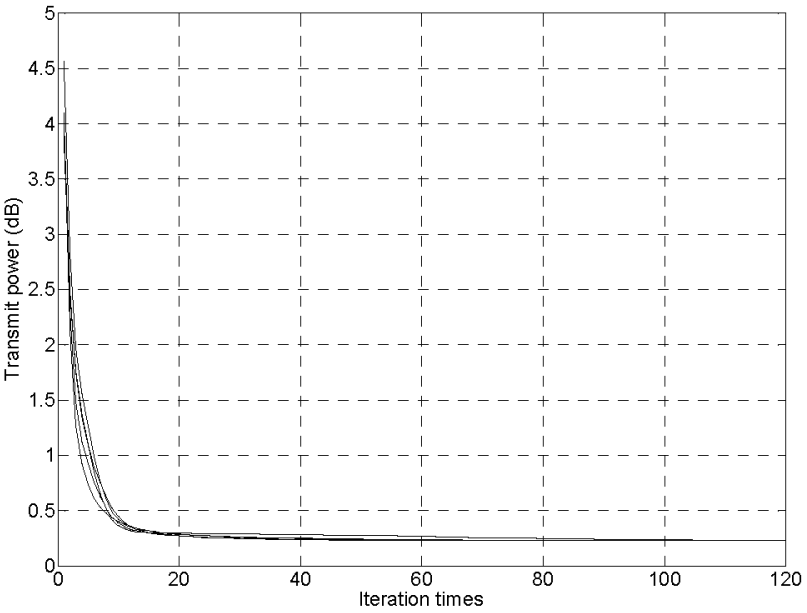

Figure 3. Convergence behavior for one channel realization.

\section{CONCLUSION}

A joint transmitter and receiver beamformers design algorithm for downlink MIMO MC-CDMA system is presented. The algorithm is iterative in nature where the transmitter beamformers and the receiver beamformers are determined alternately. The transmitter beamforming problem with a given receiver beamformer is formulated as a convex programming problem, which can be solved optimally using second order cone programming (SOCP), while the receiver beamforming problem is formulated as a constrained optimization problem and it results in a CMOE receiver. The convergence of the algorithm is analyzed and the performance of the proposed algorithm is evaluated by computing simulation.

\section{REFERENCES}

[1] S. Hara and R. Prasad, "Overview of multicarrier CDMA," IEEE Communications Magazine, vol. 35, pp. 126 - 133, Dec. 1997.

[2] A. C. McCormick and E. A Al-Susa, "Multicarrier CDMA for future generation mobile communication," Electronics \& Communication Engineering Journal, vol. 14, pp. 52-60, April. 2002.

[3] S. Hara and R. Prasad, "Design and performance of multicarrier CDMA system in frequency-selective Rayleigh fading channels," IEEE Transactions Vehicular Technology, vol. 48, pp. 1584-1595, Sept. 1999

[4] K. Kim, J. Ham, C. Lee and D. Hong, "Performance analysis of a downlink MIMO MC-CDMA system with turbo coding and channel interleaving," IEEE Vehicular Technology Conference, pp. 14391442 , Sept. 2004.

[5] D. P. Palomar, J. M. Cioffi and M. A. Lagunas, "Joint Tx-Rx Beamforimg Design for Multicarrier MIMO Channels: A Unified Framwork for Convex Optimization," IEEE Transaction on signal processing, vol. 51, pp. 2381-2401, Sept. 2003.

[6] T. M. Lok and T. F. Wong, "Transmitter and receiver optimization in multicarrier CDMA systems," IEEE Transactions on communications, vol. 48, pp. 1197-2107, July. 2000.

[7] L. M. Tuan, P. V. Su, J. Kim and G. Yoon, "An MMSE-based beamforming algorithm for smart antenna applied to an MC-CDMA system with co-channel interference," in Proc. ICCS 2002. vol. 2, pp 1252-1256, Nov. 2002.

[8] S. Boyd and L. Vandenberghe, "Convex Optimization", Cambridge University Press, 2004.

[9] W. S. Lu and A. Antoniou, "Design of Digital Filters and Filter Banks by Optimization: A state of the Art Review," in Proc. EUSIPCO'2000, vol. 1, pp. 351-354, Tampere, Finland, Sep. 2000.

[10] W. S. Lu and T. Hinamoto, "Optimal design of FIR frequencyresponse-masking filters using second-order cone programming," in Proc. ISCAS'2003, vol. 3, pp. 878-881, May 2003.

[11] H. Wolkowicz, R. Saigal and L. Vandenberge, "Handbook of Semidefinite Programming: theory, algorithms, and applications," Kluwer Academic Publishers, 2000 .

[12] J. F. Sturm, "Using SeDuMi 1.02, a MATLAB toolbox for optimization over symmetric cones," Optim. Meth. Softw., vol. 1112, pp. 625-653, 1999. 